\section{ENDOMETRITIS TUBERCULOSA}

\author{
Dr. Uriel Acero Rodríguez*
}

\section{Recuento histórico:}

Desde cuando Koch en 1882 descubrió el bacilo que lleva su nombre, muchos han sido los trabajos que se han publicado en la literatura médica sobre tuberculosis en sus distintas localizaciones. Con anterioridad, Morgani (1) en 1761 describió por primera vez un caso de tuberculosis genital, al encontrar en una autopsia de mujer joven las trompas y útero llenos de material caseoso.

Raymond en 1831 y Lisfranc en 1842 hicieron los reportes iniciales referentes a tuberculosis cérvico-uterina. Geil en 1851 la describió muy bien y es poco lo que desde entonces se ha avanzado al respecto.

\section{Frecuencia :}

Es bien sabido que la tuberculosis endometrial es secundaria generalmente a un foco tuberculoso localizado en cualquier lugar del organismo: pulmonar $80 \%$ y ganglionar $9 \%$, curada 0 en actividad.

Sutherland (2) encuentra en 65.943 ingresos habidos en el "Royal Samaritan Hospital for Women" en 20 años, 369 ( $5.6 \%$ ) casos de tuberculosis genital. De éstos, 178 (48\%) eran endometriales. El mismo autor en un grupo de 1.000 pacientes con tuberculosis pulmonar halla un $1 \%$ de afección endometrial. Sharman
(3) relata una frecuencia de $5.6 \%$, James (4) 0,1\%, González y colaboradores (5) $3.7 \%$ en 321 autopsias de enfermas con tuberculosis pulmonar. Lackner, Schiller y Tulsky (6) practicaron biopsias de endometrio en 125 mujeres con tuberculosis pulmonar y hallaron infección del endometrio sin evidencias clínicas en dos pacientes. Amaya León (7) en Colombia, en 40 pacientes con tuberculosis pulmonar avanzada, encontró 2 casos de endometritis tuberculosa.

Los bacilos de Koch pueden alcanzar los órganos genitales por vía sanguínea, linfática, peritoneal o por continuidad. De estas vías la más frecuente es la hematógena. Por lo general la tuberculosis genital ocurre después de la primoinfección entre los 12 meses en un $30 \%$, a los 3 años en un $56 \%$, a los 5 años en el $75 \%$ y con mayor frecuencia durante la adolescencia (8). El endometrio constituye la parte más afectada por la tuberculosis después de las trompas, que según la mayoría de los autores es del 100\%; para Greenberg (9) es del $45 \%$, para Petersen (10) $50 \%$, Wood y Opazo $80 \%$, Calatroni y

\footnotetext{
* Instructor del Departamento de Obstetricia y Ginecología de la Facultad de Medicina de la Universidad Nacional. - Trabajo presentado para ingresar como Socio de Número a la Sociedad Colombiana de Obstetricia y Ginecología.
} 
Ruiz (8) del 70 a $80 \%$, Amaya León (12) en la tercera parte, Botero, Montes, Acebedo y Giraldo $45.3 \%$, Valencia, Piedrahita (14) $28.5 \%$, aunque presume sea mayor este porcentaje.

\section{MATERIAL}

Quisimos hacer un estudio estadístico y clínico sobre la tuberculosis endometrial en el Servicio de Ginecología del Hospital de San Juan de Dios, de Bogotá, durante los últimos 10 años, con el fin de indagar sobre su incidencia, características clínicas, métodos de diagnóstico y tratamiento seguidos en este Servicio. Se revisaron para ello 110 historias correspondientes a los casos de tuberculosis genital que se presentaron desde Enero de 1954 hasta Noviembre de 1963; de éstas 57 (51.8\%) tenían infección endometrial y sobre ellas se basa el estudio.

La mayoría de estas pacientes, 45 (78.9\%), concurrieron al Hospital durante los últimos 4 años, aumento que seguramente no revele incremento de la tuberculosis endometrial en esta época, sino más bien a que se está investigando mejor, pues se cuenta con el funcionamiento de la clínica de Esterilidad y Tuberculosis. (Cuadro N: 1).

\section{CUADRO NN 1}

\begin{tabular}{cc}
\hline Año & $N^{0}$ de casos \\
\hline & \\
1954 & 4 \\
1955 & - \\
1956 & - \\
1957 & 5 \\
1958 & - \\
1959 & 12 \\
1961 & 9 \\
1962 & 12 \\
1963 & 12 \\
\hline
\end{tabular}

Todos estos casos fueron comprobados por exámenes histopatológicos. En total durante este período se hicieron en el Hospital 44.219 exámenes histopatológicos, de los cuales 16.707 correspondieron a piezas ginecológicas.

\section{Frecuencia por edades:}

La edad de estas enfermas osciló entre los 17 y 66 años, siendo más frecuente durante la edad activa genital. De los 22 a los 35 años se encontraron 37 pacientes con tuberculosis endometrial, o sea el $64.9 \%$, cifras similares a las encontradas por la mayoría de los autores que tratan el tema. Por encima de 50 años, se encontraron 5 pacientes, las cuales eran menopáusicas, hecho bastante raro, ya que la endometritis tuberculosa es relativamente poco frecuente en la menopausia. Así Towers (15), quien revisó la literatura mundial de 70 años halló solo 24 casos; Sutherland y Garrey (16) hallaron 7 casos entre 65.943 ingresos y Barbour (17) recoge 9 casos vistos en el "Royal Samaritan Hospital" de Glasgow en los últimos 30 años. (Cuadro №. 2).

\section{CUADRO N: 2}

\begin{tabular}{cc}
\hline Edad $\times 5$ años & $\mathbf{N}^{0}$ de casos \\
\hline $17-21$ & 2 \\
$22-25$ & 12 \\
$26-30$ & 17 \\
$31=35$ & 8 \\
$36-40$ & 6 \\
$41-45$ & 2 \\
$46-50$ & 5 \\
$51-55$ & 2 \\
$56-66$ & 3 \\
\hline
\end{tabular}

\section{Aspectos clínicos:}

El motivo de consulta fué en 18 casos $(31.5 \%)$ esterilidad: primaria de 3 a 11 años en 15 pacientes y secundaria de 6 a 7 años en 3. Ade- 
más, a pesar de que la causa de consulta no fue ésta, presentaban esterilidad primaria de 3 a 10 años 7 casos y secundaria de 4 a 11 años, 11 casos. Otros motivos de consulta fueron: (Cuadro N. 3).

\section{CUADRO № 3}

\begin{tabular}{lc}
\hline Causas de consulta & No \\
\hline Esterilidad casos \\
Irregularidades menstruales & 18 \\
Dolor abdominal & 14 \\
Prolapso genital & 11 \\
Amenorrea & 7 \\
Síntomas y signos de TBC miliar & 2 \\
Flujo vaginal & 2 \\
\hline
\end{tabular}

Irregularidades menstruales consistentes especialmente en amenorreas, oligomenorreas, polimenorreas y metrorragias, destacándose en casi todas ellas una disminución más o menos acentuada en la cantidad de sangre menstrual. Causas menos frecuentes fueron: dolor abdominal de localización baja, uni o bilateral, generalmente presente cuando existía compromiso anexial. Acusaron algomenorrea 16 pacientes, no siendo por tanto un síntoma característico tan frecuente. En 16 casos se presentó flujo vaginal, variando de aspecto según la flora asociada sin tener características especiales.

Como síntomas generales asociados, pocos fueron los anotados en las historias, debido talvez a falta de un interrogatorio apropiado. Elevaciones de temperatura vesperales, sudores nocturnos, taquicardia, astenia, anorexia, enflaquecimiento, anemia, fueron infrecuentes; se encontraron en 12 casos, en los cuales había concomitancia con tuberculosis pulmonar o generalizada. Al examen ginecológico los hallazgos encontrados, en aquellas pacientes con endometritis tuberculosa sin lesiones asociadas, no descubrió nada llamativo.
En 18 pacientes se encontraron datos en anamnesis personal o familiar que indicaban o hacían sospechar infección tuberculosa, hecho importante $y$ de gran ayuda para el diagnóstico.

En resumen, la infertilidad fué el síntoma dominante (Cuadro No 4) y como lo anotamos anteriormente, estuvo presente en la serie revisada en 36 casos $(63 \%)$, cifra semejante a la de otros autores, como Sánchez Contreras y García Medrano (18), quienes encontraron un $76 \%$.

CUADRO NN 4

FRECUENCIA DE ESTERILIDAD E INFERTILIDAD

\begin{tabular}{cc}
\hline Esterilidad primaria & Esterilidad secundaria \\
\hline $\begin{array}{c}22 \\
\text { De } 311 \text { años }\end{array}$ & $\begin{array}{c}14 \\
\text { Abortos } \\
6\end{array}$ \\
Normales \\
En No de 1 a 6 & Multiparidad \\
\hline
\end{tabular}

Diversas estadísticas anotan entre las causas totales de esterilidad la tuberculosis genital, como responsable en un 4 a $7 \%$. De Carle (19) en estudio sobre 201 casos, halló un $30 \%$ de esterilidad. Sharman (3) en 3.804 casos de infertilidad primaria, estudiados en 20 años, encontró 216 ca$\operatorname{sos}(5.6 \%)$ debidos a tuberculosis endometrial.

En 14 casos de los aquí estudiados, la única manifestación resultó ser la esterilidad y en 4 de éstos, había bloqueo tubario bilateral demostrado por Histerosalpinografía; en el resto de los casos no se practicó este examen. Sin embargo, es de suponer que la esterilidad no se pueda imputar a una alteración endometrial exclusiva. En Palestina donde parece estar muy diseminada esta enfermedad, Rabau (20) descubrió tuberculosis del endometrio en 
el $3.5 \%$ de 2.000 mujeres estériles; en el $25 \%$ de estos casos las trompas se hallaban permeables, por lo cual para este autor la lesión endometrial constituye una causa absoluta de esterilidad. Richard TeLinde, (21) cree que esta aseveración es infundada, ya que lo que sucede es que seguramente en todos los casos existía salpingitis tuberculosa y este trastorno infeccioso de las trompas interfiere en la migración del huevo, aun cuando la prueba de Rubin demuestre permeabilidad tubaria.

La amenorrea y demás alteraciones menstruales, manifestaciones en algunos casos de tuberculosis endometrial, se explican por la destrucción del endometrio parcial o totalmente. De acuerdo con el grado de lesión endometrial se presentan los diferentes cambios en el ciclo menstrual. DiPaola (22) observa dos tipos de lesiones endometriales: una folicular, en la cual el endometrio funciona normalmente y otra caseosa, en la que hay amenorrea por destrucción tisular. Amaya León y Cols. (23) creen sin embargo, que la amenorrea es debida a un fenómeno general y no a lesión local.

En 6 casos se presentaron abortos, así: en cuatro pacientes 1 aborto, en una 2 y en la restante 6 , ésta había tenido anteriormente 5 embarazos de caracteres normales. Tales abortos pudieron ser debidos a que ya existía la tuberculosis endometrial - a que ésta seguramente desencadenó el aborto.

El cuadro hemático y la eritrosedimentación demostraron normalidad en la mayoría de los casos de tuberculosis endometrial sin lesiones asociadas, por lo tanto, es un dato que adquiere mayor valor cuando hay concomitancia con lesiones anexiales, pulmonares, del peritoneo 0 en la tuberculosis miliar, en donde siempre se encontró un aumento de los linfocitos que osciló entre 35 y $54 \%$. La eritrosedimentación en los mismos casos estuvo aumentada en cifras que variaron entre 16 y 110 $\mathrm{mm}$.

\section{Métodos de diagnóstico}

La biopsia de endometrio resultó ser el método más usado para el diagnóstico de la tuberculosis endometrial; en 43 casos se hizo por este procedimiento $(75.4 \%)$; por raspado uterino en 3 casos; hallazgos quirúrgicos en histerectomías vaginales en pacientes que consultaron por prolapso genital: 4 y 2 en histerectomías totales abdominales por miomatosis uterina; hallazgos de autopsia en 4 pacientes, las cuales murieron por tuberculosis miliar.

\section{CUADRO NN 5}

\begin{tabular}{lc}
\hline Método de diagnóstico & $N^{0}$ de casos \\
\hline Biopsia endometrial & 43 \\
Raspado uterino & 3 \\
Hallazgo quirúrgico & 6 \\
Hallazgo de autopsia & 4 \\
\hline
\end{tabular}

Las biopsias fueron practicadas en la mayoría de los casos en el curso del estudio de esterilidad. Su valor es relativo, ya que el resultado negativo no descarta la presencia de la infección tuberculosa. James (4) anota que se deben hacer varias tomas $3 \circ 4$ días después de la regla, de preferencia biopsias fúndicas $y$ repetirlas en ciclos consecutivos en caso de respuesta negativa. Otros y entre ellos nosotros, recomendamos tomar la biopsia en el período premenstrual.

O'Brien y Lawlor (24), declaran haber descubierto tuberculosis del endometrio en 37 biopsias realizadas a 23 pacientes durante un perío- 
do de $8 \frac{1}{2}$ años, todas ellas en mujeres exentas de evidencia clínica de la enfermedad. En 17 de estas pacientes, la alteración más importante era la esterilidad.

Fuster (25), después de haber estudiado el material procedente de 243 tuberculosis genitales por biopsia endometrial, biopsia de endocérvix y de cuello, reconoce que quizá alrededor de un $12 \%$ de tuberculosis no se consigue demostrar por este procedimiento.

La biopsia podría exacerbar o producir diseminación hematógena, cosa improbable si se tienen los cuidados necesarios para evitarla. En un caso dei presente estudio, hubo reacción anexial intlamatoria unilateral después de practicada la biopsia.

El raspado uterino es más eficiente para el diagnóstico positivo y se vuelve indispensable en aquellos casos sospechosos de tuberculosis endometrial y en los cuales la biopsia ha sido negativa. Debe ser completo y realizado con cautela para evitar exacerbaciones o reactivación de un proceso anexo-peritoneal hasta el momento asintomático. $\mathrm{Si}$ existen procesos inflamatorios, el raspado debe hacerse únicamente si la temperatura, el leucograma y la eritrosedimentación revelan que el proceso anexial no está en actividad. Algunos aconsejan además, reposo por tres días después del raspado y el uso de antibióticos, tipo Penicilina, asociado con Estreptomicina, por el mismo tiempo como mínimo.

En este estudio solamente se hicieron raspados uterinos en 7 pacientes. En un caso con fin diagnóstico en esterilidad, en otro como tiempo previo a Operación de Manchester, er, otro con fin terapéutico y diaanóstico por flujo sanguinolen- to de larga evolución y en los cuatro restantes como controles durante el tratamiento tuberculostático.

Otros métodos coadyuvantes en el diagnóstico de la tuberculosis endometrial son: 1? Baciloscopia: se observan directamente los bacilos por medio de la tinción de Ziehl-Nilsen. 2. Cultivo del bacilo a partir de la sangre menstrual. 3 Cultivo del material endometrial obtenido en biopsias y raspados. 4\% Inoculación al curí o al cobayo. 5․ Histerosalpingografía. 6: Reacciones tuberculínicas.

En nuestro estudio, la baciloscopia se practicó en la mayoría de los casos, pero desafortunadamente, no todos los protocolos se adjuntaron en las historias clínicas; se encontraron 8 casos positivos, 6 negativos $y$ en 5 no había llegado el resultado en el momento de la revisión. El cultivo de la sangre menstrual y del material endometrial se hizo en 16 casos con resultados positivos en 4 , colonias sospechosas en 3 y negativos en el resto. Este método requiere procedimientos especiales muy dispendiosos para llegar a ser efectivo $\checkmark$ el resultado solo se valora de 2 a 6 semanas después. Amaya León y colaboradores (23) en su trabajo sobre tuberculosis genital, da resultados más específicos sobre este procedimiento.

La inoculación al curí no se hizo en ningún caso; es un método muy sensible y sirve cuando los otros métodos no han detectado la infección. Rodríguez H. (26) aconseja la inoculación de secreción menstiual al curí y afirma que este método sencillo e inocuo, es tan fiel como la biopsia del endometrio para descubrir la tuberculosis endometrial y superior a ella en la investigación de tuberculosis genital latente y anexial. 
La histerosalpingografía se practicó solamente en 7 casos. En 5 demostró bloqueo tubario bilateral. Se describen imágenes típicas en los estudios radiográficos de la cavidad uterina, las cuales según la mayoría de los autores, son: desproporción entre el cuello y la cavidad uterina; úteros deformados, irregulares y empequeñecidos radiográficamente, laterodesviación del cuerpo uterino, dentelado como de sierra que se observa en el canal cervical o en la superficie del endometrio. Sin embargo, no se puede hablar de imágenes patognomónicas, sino sospechosas, que de acuerdo con la clínica y demás exámenes de laboratorio ayudan a un mejor diagnóstico de la enfermedad.

Basch y Pellerin (27), dicen que este método y la biopsia son los elementos más importantes y decisivos en el diagnóstico de la tuberculosis genital.

Las reacciones tuberculínicas no se practicaron, son inseguras y tan solo tienen valor cuando son negativas.

Surany, Cavaller y Berenecsi (28) recomiendan para el diagnóstico de la tuberculosis genital, cultivos en serie de la secreción intermenstrual por un procedimiento que ellos describen claramente en un trabajo sobre 72 casos; la exploración bacteriológica de los cultivos de la secreción intermenstrual, concluyen los autores, complementan y mejoran los resultados del estudio histológico.

\section{Lesiones genitales asociadas}

Se encontraron en el presente trabajo endometritis tuberculosa sin lesiones asociadas a otros órganos en 27 casos ( $47.3 \%)$; concomitancia con tuberculosis de cérvix en 5; en anexos 24; en peritoneo 10; y en ovarios 5. (Cuadro N. 6).

\section{CUADRO N: 6}

\begin{tabular}{lc} 
Localización de la TBC & $\mathbf{N}^{0}$ de casos \\
\hline Endometrio únicamente & 27 \\
Endometrio & 13 \\
Anexos & \\
Endometrio & 4 \\
Cérvix & \\
Endometrio & \\
Anexos & 5 \\
Peritoneo & \\
Endometrio & \\
Anexos & \\
Peritoneo & \\
Cérvix & \\
Endometrio & 1 \\
Anexos & \\
Peritoneo & \\
Ovarios & \\
Endometrio & \\
Anexos & \\
Ovarios & \\
Miliar & \\
\hline
\end{tabular}

\section{Asociación con lesiones pulmonares}

Se investigó tuberculosis pulmonar en 47 pacientes, con fotofluorografía, radiografía de tórax en placas $14 \times 17$ o investigación de BK en el esputo. (Cuadro N. 7).

En 34 pacientes los exámenes fueron negativos; en 3 también negativos, pero había evidencia en la radiografía de lesiones residuales; en una había tuberculosis fibronodular inactiva; en otra, paquipleuritis posiblemente tuberculosa; en otra se encontraron lesiones avanzadas activas de tuberculosis y BK en esputo, positivo; en 7 se hallaron signos de lesiones de tuberculosis pulmonar activa. No se hizo investigación pulmonar alguna en 6 casos, de los cuales, uno con historia sospechosa de tuberculosis pulmonar. A una paciente con lesión residual parenquimatosa, en quien los exámenes practicados: fotofluorografía, radiografía de tórax e investigación de BK en el esputo, resultaron negativos para tuberculosis en actividad, se le hizo tratamien- 
CUADRO № 7

\begin{tabular}{|c|c|c|}
\hline Métodos de investigación & $\mathrm{N}^{\mathbf{0}}$ de casos & Lesión pulmonar \\
\hline Fotofluorografía & 16 & No \\
\hline $\begin{array}{l}\text { Fotofluorografía } \\
\text { Radiografía de Tórax }\end{array}$ & 15 & No \\
\hline $\begin{array}{l}\text { Fotofluorografía } \\
\text { Radiografía de Tórax } \\
\text { Esputo: Inv. BK }\end{array}$ & 2 & No \\
\hline $\begin{array}{l}\text { Fotofluorografía } \\
\text { Esputo: Inv. BK }\end{array}$ & 1 & No \\
\hline Fotofluorografía & 1 & Lesión residual pleural \\
\hline $\begin{array}{l}\text { Fotofluorografía } \\
\text { Radiografía de Tórax } \\
\text { Esputo: Inv. BK }\end{array}$ & 2 & Lesión residual parenquimatosa \\
\hline Radiografía de Tórax & 1 & $\begin{array}{l}\text { TBC fibronodular inactiva. } \\
\text { Paquipleuritis }\end{array}$ \\
\hline $\begin{array}{l}\text { Radiografía de Tórax } \\
\text { Esputo: } \mathrm{BX}+\end{array}$ & 1 & Lesiones cavitarias avanzadas \\
\hline Radiografía de Tórax & 7 & TBC activa \\
\hline En estudio, sin llegar exámenes & 4 & \\
\hline No investigadas & 6 & \\
\hline
\end{tabular}

to médico únicamente durante el tiempo que estuvo hospitalizada ( 20 días), con diagnóstico de endometritis tuberculosa y anexitis bilateral; al salir del Hospital, las masas anexiales habían desaparecido y el estado general era satisfactorio; la enferma no volvió a control; 19 meses más tarde, regresó al Hospital consultando por síntomas pulmonares y murió el mismo día en edema agudo pulmonar, debido a tuberculosis avanzada.

\section{Tratamiento}

La mayoría de los autores están de acuerdo en que el tratamiento se debe hacer con Estreptomicina y un tuberculostático; algunos utilizan Acido Paramino-salicílico (PAS), otros Para-amino-salicilato de isonicotil-hidrazida (PIEM), otros Isoniazida; re- portando todos resultados más o menos satisfactorios. El objetivo del tratamiento es obtener una respuesta terapéutica favorable con la mayor rapidez posible, sin provocar reacciones tóxicas o resistencia y evitar episodios de irritación vestibular irreversibles, con la aplicación de la Estreptomicina.

Para la aplicación de la Estreptomicina, TeLinde (21) aconseja tener en cuenta los siguientes factores: 1? Estado general de la paciente, incluyendo temperatura, peso, anorexia y duración de la enfermedad. 2\% Extensión de la lesión tuberculosa genital y lesiones asociadas en pulmones, riñones, huesos, etc. 3! Función renal, ya que el $80 \%$ de la Estreptomicina administrada, deberá ser excretada por el riñón normal en 24 
horas, por lo cual cualquier trastorno en esta función puede exponer a la paciente a reacciones tóxicas graves. 4: Sensibilidad del organismo al medicamento, indicada por la respuesta clínica a las drogas.

En la serie revisada se hizo tratamiento médico a 42 pacientes, consistente en la mayoría de ellas, en Estreptomicina o Dihidroestreptomicina y un tuberculostático, casi siempre Para - amino - salicilato - isonicotil - hidrazida, en otros casos Acido Paramino-salicílico e Hidrazida del ácido isonicotínico, durante un tiempo variable, en general muy corto, además de irregular porque las pacientes no asistieron debidamente a los controles. En cuatro casos no se hizo tratamiento médico por muerte de la paciente a las pocas horas de ingreso al Hospital, por tuberculosis miliar. En los restantes casos, no se practicó tratamiento debido a que cuando se hizo el diagnóstico de endometritis tuberculosa, las pacientes no volvieron al Hospital. Continúan en tratamiento y control 23 pacientes. Es indispensable insistir en hacer comprender a las pacientes la importancia de su padecimiento y de las características de éste, para lograr un mejor y más eficiente tratamiento; ya que buena parte de las pacientes, como lo anotamos anteriormente, no volvieron al Hospital, en especial aquellas que han consultado por esterilidad y se enteraron de las pocas probabilidades de éxito en cuanto a fecundidad se refiere y vieron así defraudadas sus esperanzas de llegar a embarazarse.

El tratamiento quirúrgico es discutido por varios autores $y$ en general hay acuerdo con respecto a cuándo y cómo debe hacerse. TeLinde (21) es de opinión de que en caso de comprobar tuberculosis extensa del endometrio, conviene recurrir a tratamien- to quirúrgico, luego de tratamiento médico usual, siempre que se demuestre en los controles la no mejoría; de lo contrario, es preferible continuar el tratamiento médico. Foix (29) considera tributarios de la cirugía, aquellos casos de endometritis tuberculosa que persisten a pesar del tratamiento, aún cuando no se toquen masas anexiales, pues la experiencia demuestra que al operar a estas enfermas, se encuentran lesiones tubarias. La cirugía se debe adaptar a cada paciente, teniendo en cuenta edad y paridad para ser conservador o radical. Schaeffer (30) dice que cuando está indicada la cirugía en tuberculosis genital, lo mejor sería la aplicación de una terapia antimicrobiana preoperatoria por espacio de tres a cuatro meses para seguir, después de la cirugía, con la quimioterapia por espacio de un año más. Foix (29) anota aue, si por error diagnóstico al efectuar una laparotomía nos encontramos con una tuberculosis genital, lo aconsejable es cerrar el abdomen, someter a la paciente a tratamiento antibacteriano y en el momento oportuno, efectuar la exéresis. Proceder en sentido contrario sería retroradar a la era preantibiótcia, con graves riesgos para la paciente. Después del tratamiento medicamentoso parece que disminuye el edema y hay neta mejoría del endurecimiento tisular, por lo tanto, la intervención resulta técnicamente menos difícil y evita la gran morbilidad que sin ésta puede acarrear.

No debe practicarse cirugía reparadora en tuberculosis confirmada, ya que los embarazos obtenidos no se deben a la intervención plástica, sino al tratamiento médico. Schaeffer (30) comprobó que en casos en los cuales parecía lograda la curación del endometrio y trompas con terapéutica antituberculosa, las trompas 
extirpadas mostraban tal desorganización, que los órganos carecían de función. Por lo tanto, es evidente que las operaciones plásticas carecen de valor.

En nuestro trabajo las operaciones practicadas no se hicieron con diagnóstico previo de tuberculosis, por lo cual ol tratamiento quirúrgico llevado a cabo fué determinado por afecciones orgánicas diferentes a la entidad que nos ocupa; el diagnóstico fue hallazgo quirúrgico $\circ$ histológi$\mathrm{co}^{\text {; }}$ on algunas de estas pacientes se hizo tratamiento post-operatorio por tiempo variable.

La tendencia actual es prolongar el tratamiento médico por lo menos dos años. Foix (29) aconseja un gramo de Estreptomicina cada dos o tres días y 5 mgrs. de Hidrazida del ácido isonicotínico por kilogramo de peso y por día ,por el tiempo necesario, de acuerdo con cada caso clínico. Varios autores, consideran que hay curación de la tuberculosis endometrial con tratamiento médico bien dirigido, con dosis variables de Estreptomicina, que oscilan entre los 50 y 90 gramos, en un 83 a $86 \%$; otros, entre ellos Bunster (31) consigue el $100 \%$ de curación endometrial con tratamiento de Estreptomicina y la Hidrazida del ácido isonicotínico durante un tiempo prolongado de 210 días; Wood y Elgueta (32) declaran que no hay en el presente un tratamiento que garantice el éxito completo en la curación de la tuberculosis genital.

El tratamiento usado en la actualidad en el Servicio de Ginecología del Hospital San Juan de Dios, es el siguiente: tratamiento médico en todas las pacientes con Estreptomicina, 1 gr. I.M. diario y Para-amino-salicilato de isonicotil-hidrazida en comprimidos, en dosis de 15 a 20 mgrs. por kilogramo de peso, por tres meses, agregando Piridoxina 500 mgrs. para evitar toxicidad, luego se reduce la Estreptomicina a 2 grms. semanales, continuando en esta forma por el tiempo requerido de acuerdo a la evolución clínica de cada caso, por un tiempo mínimo de dos años.

El tratamiento quirúrgico, como dijimos anteriormente, no se ha hecho, pero en los casos que continúan en control, se hará teniendo en cuenta los conceptos antes mencionados, precedidos de tratamiento médico por un tiempo mínimo de 3 a 4 meses.

Cualquiera que sea el tratamiento empleado, no se considerará curada a una enferma de tuberculosis endometrial hasta tanto haya transcurrido por lo menos 3 años, con exámenes de control durante este tiempo, de acuerdo con la evolución clínica (exámenes ginecológicos, hemogramas, eritrosedimentación, cultivos, inoculaciones, etc.). Debe tenerse presente que una o dos biopsias, o inoculaciones negativas no significan que la enferma esté curada y que pueda embarazarse.

\section{Controles y resultados}

De las 42 pacientes a quienes se les hizo tratamiento médico, a 17 se les practicó biopsias endometriales de control en tiempo que osciló entre los 4 y 8 meses de tratamiento, continuando positivas para tuberculosis en 15 y negativas en 2. De las primeras enfermas, 4 abandonaron el tratamiento a pesar de la positividad del examen. A una se le hizo raspado uterino y biopsia endometrial, persistiendo la afección después de 4 y medio años de tratamiento irregular; 6 meses más tarde una nueva biopsia fué negativa, lo mismo que otro control biópsico 3 años después. A 5 enfermas se les hizo 
raspado uterino después de $3,4,10$, 12 y 36 meses de tratamiento, mostrando ausencia de tuberculosis endometrial. De las 6 pacientes a quienes se les hizo el diagnóstico por hallazgo histológico, después de ser histerectomizadas, dos continúan en tratamiento y el resto asistieron al Hospital únicamente en dos o tres ocasiones más y luego se perdieron de vista. Las 14 enfermas restantes se encuentran asistiendo debidamente a la Consulta de Tuberculosis Ginecológica, junto con nueve de las pacientes antes mencionadas.

Respecto a las menstruaciones, el resultado del tratamiento es difícil de valorar debido a la falta de anotaciones a ese respecto en las historias clínicas. De las 4 pacientes que presentaban amenorrea, se controlaron 3 , en 2 no hubo mejoría y en la tercera, a los 7 meses de tratamiento, se iniciaron menstruaciones irregulares en escasa cantidad. Sutherland (2) de 18 pacientes controladas con amenorrea, encuentra 6 en las cuales no hubo mejoría y 12 que presentaron reglas normales con el tratamiento.

Respecto a embarazos, se obtuvieron tres. Uno a los 27 meses de hecho el diagnóstico, regresó la paciente con un embarazo ectópico derecho roto; había sido tratada durante un año y los exámenes de control eran negativos para tuberculosis. Otra paciente llevaba 3 meses de tratamiento para tuberculosis anexial y peritoneal, diagnóstico hecho cuando tenía aproximadamente 5 semanas de embarazo, por laparotomía; posteriormente abortó. Un mes más tarde la biopsia de control resultó positiva para endometritis tuberculosa. La tercera paciente a los 7 meses de iniciado el tratamiento quedó embarazada, desafortunadamente, la pa- ciente no volvió al hospital desde cuando tenía 3 meses de gestación.

Según autores israelitas, pocas veces podrá producirse el embarazo ein pacientes con endometritis tuberculosa. Herring y King (33), presentaron un caso de mujer embarazada que dio a luz un niño que falleció a los 22 días, a causa de tuberculosis generalizada; la biopsia de endometrio y cérvix demostró tuberculosis.

Varios autores que han estudiado el problema de tuberculosis genital y embarazo en sus diferentes países, han publicado en la literatura médica los embarazos logrados después de un tratamiento tuberculostático adecuado, son relativamente muy pocos. De los trabajos revisados hemos encontrado 68 embarazos. Entre ellos, Reyden (34) en Suecia, en una serie de 2.118 pacientes con tuberculosis genital, con tratamiento médico, consigue el $2.2 \%$ de embarazos a término, $0.98 \%$ de ectópicos y el $0.56 \%$ de abortos. En Colombia Botero y Restrepo (35) publicaron dos casos de tuberculosis endometrial, uno con embarazo concomitante que progresó hasta el $7^{\circ}$ mes y otro que se embarazó luego de tratamiento tuberculostático apropiado. Amaya León (23) reporta otro caso con endometritis tuberculosa tratada, logrando un embarazo que termina por aborto de 10 semanas. Helbrecht (36) afirma, después de una cuidadosa revisión de 100 casos sobre los embarazos en pacientes con tuberculosis genital tratada, que cualquier embarazo que se produzca requiere vigilancia muy cuidadosa, considerando que tal embarazo tiene cuatro posibilidades sobre una de ser tubárico o de terminar en aborto.

\section{Sumario y conclusiones}

1 . Se hace un estudio estadístico y clínico sobre tuberculosis endome- 
trial, de las pacientes que acudieron en los últimos 10 años, al Servicio de Ginecología del Hospital de San Juan de Dios de Bogotá.

2? Se revisan 110 historias, correspondientes a los casos de tuberculosis genital en este período, de las cuales 57 tenían infección endometrial y sobre ella se basa este estudio, siendo comprobados todos los casos por exámenes histo-patológicos.

3. La tuberculosis endometrial es frecuente en Colombia y es causante de un buen número de infertilidades.

4. La edad activa genital es la más afectada por la infección. Sin embargo, se encontraron 5 pacientes postmenopáusicas, cifra proporcionalmente alta para el resto de pacientes.

5. Se piensa muy poco en esta enfermedad y por ello se hace una historia clínica deficiente, en cuanto se refiere a anemnesis personal y familiar.

6: La infertilidad es el síntoma dominante; la amenorrea y las irregularidades menstruales son poco frecuentes.

7: No se encontraron hallazgos especiales al examen ginecológico, en las pacientes con endometritis tuberculosa sin lesiones asociadas.

8. La biopsia endometrial fue el método más usado para el diagnóstico. El raspado uterino se hace indispensable para aquellos casos sospechosos de infección endometrial. Orros métodos diagnósticos no se hicieron de rutina en las pacientes estudiaclas.

9: Se encontró tuberculosis endometrial sola, en el $47.3 \%$ de los casos. Concomitancia con tuberculosis anexial en el $42.1 \%$, cervical en el $8.7 \%$, peritoneal en el $17.5 \%$ y ová- rica en el $8.7 \%$. Asociación con lesiones pulmonares activas o residuales en el $22.8 \%$.

10. El tratamiento en la mayoría de las pacientes consistió en Estreptomicina y Para-amino-salicilato de isonicotil-hidrazida, en general deficiente por falta de colaboración de las pacientes.

11. No se hizo tratamiento quirúrgico con fin terapéutico; las operaciones practicadas se hicieron sin diagnóstico previo de tuberculosis.

12. Se obtuvieron 3 embarazos en las pacientes controladas: uno ectópico, otro que terminó en aborto y el tercero ortotópico.

13. Es necesario una mayor investigación de la tuberculosis genital y de tratar de hacer comprender a las pacientes la importancia del tratamiento y por ende de la necesidad de la debida asistencia a los controles, para así mismo obtener los mejores resultados en la lucha contra esta enfermedad.

\section{BIBLIOGRAFIA}

1 MORGAGNI, G. B.: Citado por Lemperer, P.: The pathology of Morgagni an Virchow. Bull. Hist. Med., $32: 24,1958$.

2 SUTHERLAND, A. M.: Tuberculosis of endometrium. J. Obst. Gyn. Brit. Empire, 63: $161,1956$.

3 SHARMAN, A.: Endometrial tuberculosis in Sterility. Fertil Steril, 3:144, 1952.

4 JAMES; M. YANEVA, H.: Formes pauxinodulaires de tuberculose endometriale. Bull. Fed. Soc. Gyn. Obst. Fr. 8:587, 1956.

5 GONZALEZ, A. A. y cols.: Tuberculosis pulmonar, estudio de 321 casos de autopsias. Prensa Médica Mexicana. 24:131, 1959.

6 LANHNER, J. E., SCHILLER, E. y TULSKY, A. S.: Coincidence of tuberculosis of the endometrium with tuberculosis of the lung, Am. J. Obst. \& Gynec. 40:429, 1940. 
7 AMAYA LEON H., CAMERO R., GOMEZ V.: Endometrio tuberculoso. Memorias de la II Convención Colombiana de Obst. y Ginec. Cali : 249, 1955.

8 CALATRONI, C. . y RUIZ, V.: Terapéutica Ginecológica, $40: 850$, 1958 .

9 GREENBERG, J. P.: A clinical study of tuberculous salpingitis, based on 200 cases, Bull. ohns Hopkins Hosp., 32:72, 1921.

10 PETERSEN, REUBEN: Pelvic tuberculosis; en results of operative treatment Am. J. Obst. \& Gynec. 4\% 234, 1922.

11 WOOD y OPAZO: Revista Latinoamericana Obs. y Ginec., Ag. Sep., 1953.

12 AMAYA LEON, H.: Rev. Fac. Méd. Bog Col., 1949

13 BOTERO P., MONTES S., ACEBEDO F., GIRALDO G.: Tuberculosis genital femenina. Rev. Col. Obst. y Ginec. XV: 137, 1964.

14 VALENCIA PIEDRAHITA: Tuberculosis genital en la mujer. Rev. Col. Obst. y Ginec. VII: 69, 1955.

15 TOWERS, R. P.: Post Menopausal endometrial Tuberculosis: an unusual case with a review of previous reports, J. Obst. Brit. Empire, 61: 657, 1954

16 SUTHERLAND, A. M. y GARREY, M. M. : Feimale genital tuberculosis: Twenty year clinicai survey, Glasgow, M. J., 32: 231, 1951.

17 BARBOUR, E. M.: Postmenopausal endometrial Tuberculosis. J. Ohat. Brit. Empire, 67: 1008-1062, 1961.

18 SANCHEZ CONTRERAS J. Y GARCIA MEDRANO P.: Tuberculosis del endometrio, estudio anatómico y c'ínico. Rev. de Obst. y Ginec. de México, 26: 209, 1961.

19 DE CARLE, D. W.: Pelvic tuberculosis, It's relationship to sterility, present and future, Fertil \& Steril. 6: 534, 1955.

20 RABAU, ERWIN: Genital tuberculosis of female and sterility, Fertil \& Steril, 1: $517,1950$.

21 TELINDE, RICHARD W': Ginecología operatoria. Segunda Ed. 30: 589, Edit. Bernades, Buenos Aires, 1961.

22 DIPAOLA, G.: Endometritis tuberculosa y alteraciones menstruales. Obst. y Ginec. Latino Americanas, 12: 147, 1954.
23 AMAYA LEON, H. y cols.: Tuberculosis genital femenina. Estudio clínico, diagnóstico y estadístico en Bogotá, Colombia. Rev. Col. Obst. y Ginec. XV: 111, 1964.

24 O'BRIEN, J. R. P. y LAWLOR, M. P. : Unsuspected tuberculous endometritis, J. Obst. \& Gynec. Emp. 54: 636, 1947. Citado por TeLinde, Richard W.

25 FUSTER, R.: Tuberculosis genital. Progresos en Obstetricia y Ginecología. 4: 198, 1961.

26 RODRIGUEZ G. H.: La inoculación de secreción menstrual al cobayo en el diagnóstico de tuberculosis genital. Rev. Col. Obst. y Ginec. X: 152, 1959.

27 BASCH, M., PELLERIN, M.: Trois observation d'endometrite tuberculeuse, Compts rendus de la Société Fr. gyn. 26: 114, 1956.

28 SURANY, S., CAVALLER, I., BERENECSI, G., Diagnostik der Genital tuberkulose der Frau. Zeitschrift für Geburtshilfe und Gynakologie, 150: 90-103, 1958. Citado por Dexeus.

29 FOIX, A.: La cirugía en la tuberculosis genital. Obst. y Ginec. Latino Americanas, 20: $159,1962$.

30 SCHAEFFER, G.: Diagnosis and treatment of female tuberculosis. Clin. Obst. \& Gynec. 2: 530. 1959

31 BUNSTER, E. M.: Trompa de Falopio; esterilidad de causa tubaria. Edit. Guillermo Kraft Ltda. Buenos Aires, 1951.

32 WOOD, J., ELGUETA, H.: Tuberculosis genital y esterilidad. Ginec. Prat. 9: 153, 1958.

33 HERRING y KING: Ginecología operatoria, segunda Edic. 30: 589, Edit. Bernades, Buenos Aires, 1961. Citado por TeLinde, Richard W.

34 REYDEN : Role of histerosalpingography in the diagnosis of genital tuberculosis in women. Ann. Chir. Ginec. Femm. 44: 7 , 1955. Citado por Ilinen y Johanson.

35 BOTERO U., RESTREPO S.: Tuberculosis endometrial y Embarazo. Rev. Col. Obst. y Ginec. XII: 211, 1961 .

36 HALBRECHT, L., TIQUA P.: The value of culture and endometrial biopsy in the diagnosis of genital tuberculosis. A.m. J. Obst. 75: 899. 1958. 